\title{
How to Measure the Kinetic Energy Distribution of the Precursor Ion Main Beam in Mass-Analyzed Ion Kinetic Energy Spectrometry Experiments
}

\author{
Fred H. Strobel and Jeanette Adams \\ Department of Chemistry and the Emory University Mass Spectrometry Center, Emory University, Atlanta, \\ Georgia, USA
}

\begin{abstract}
Scans of the electrostatic analyzer (ESA) across the precursor ion beam in reverse-geometry (BE) mass spectrometers that are operated under double-focusing conditions do not measure the "energy resolution of the main beam": They only measure double-focusing resolution. The only way that ESA scans can measure the kinetic energy distribution of the main beam is to operate the instrument so that angular (directional) focusing is not achieved. Thus, the mass spectrometer is no longer double-focusing. Under double-focusing conditions, however, scans of the accelerating voltage while the magnetic field and ESA are held constant can be used to measure either the kinetic energy distribution of the main beam that enters the magnet or the energy-resolving power of the instrument. Scans at a constant ratio of $B^{2} / E$ can be used similarly. The energy-resolving power of any ESA is defined by its dispersion and the widths of the energy-resolving object and image slits that immediately precede and follow the ESA, respectively. The use of $B E, E B$, and triple-sector instruments to measure energy-resolving power and the kinetic energy distribution of the precursor ion main beam is compared and discussed. (J Am Soc Mass Spectrom 1995, 6, 1232-1242)
\end{abstract}

$\mathrm{R}$ everse-geometry $(\mathrm{BE}$, in which $\mathrm{B}$ is the magnetic analyzer and $E$ is the electrostatic analyzer or ESA) mass spectrometers have been used for mass-analyzed ion kinetic energy spectrometry (MIKES) experiments for approximately 20 years [1]. The magnet is used to mass-select the precursor ion main beam, which is transmitted to the second fieldfree region between $B$ and $E$. Products of metastable ion decompositions are observed by scans of the ESA. The shapes of the product ion peaks may be used to determine the kinetic energy release distributions (KERDs) of the metastable ion reactions.

Researchers traditionally have reported a parameter termed the "energy resolution of the main beam," which is defined as " $E / \Delta E$ ", by scans of the ESA across the precursor ion beam [1-9]. The major assumption is that ESA scans measure the kinetic energy distribution of the main beam. Consequently, the $\Delta E$ term in $E / \Delta E$ is assumed to be equal to the measured "kinetic energy width" of the precursor ion beam. The kinetic energy distribution of the main beam $(\Delta E)$ must be small to guarantee that it does not contribute significantly to the measurement of the KERD. To ensure that $\Delta E$ is small, it must be measured correctly.

Address reprint requests to Professor Jeanette Adams, Department of Chemistry, Emory University, Atlanta, GA 30322.
There is one important problem in this traditional method used to measure $\Delta E$ : The technique is not in accord with principles of velocity and directional focusing in double-focusing mass spectrometers as established 60 years ago by Herzog [10] and later by Bainbridge and Jordan [11], Johnson and Nier [12], Hintenberger and König [13], and Barber et al. [14]. In any double-focusing arrangement of sector fields, the velocity dispersion of the magnet is opposite and approximately equal to the velocity dispersion of the ESA. Thus, for an ion beam of a particular mass-tocharge ratio formed in the ion source of a $B E$ instrument, the spread of velocities and thus kinetic energies $(\Delta E)$ is dispersed and directionally focused by the magnet but then refocused to one point by the ESA. Consequently, under double-focusing conditions, it is impossible for ESA scans to show the kinetic energy (velocity) distribution of the precursor ion beam. A similar argument is also true for forward-geometry (EB) instruments.

Scans of the ESA of any double-focusing mass spectrometer (BE or EB) across a precursor ion beam formed in the ion source measure double-focusing mass resolution. This fact was established by Swann [15] and Bleakney [16] in the 1930s. Furthermore, this fact is the basis for the widely used mass-doublet peak-matching technique established for double-focusing mass spectrometers by Nier and co-workers [17] and Barber et al. 
[14], who determined the accurate mass ratio of $\mathrm{H}_{2}^{+} / \mathrm{D}^{+}[17 \mathrm{~b}]$ and other ions by using ESA scans.

Another important aspect of measurement of the "energy resolution of the main beam" is the effect of energy-resolving slits on energy-resolving power. The energy-resolving power of an ESA (or magnet) of a mass spectrometer is defined by its dispersion and the widths of both the object and image slits that immediately precede and follow the ESA (or magnet), respectively. This is clear from eq 1 , which defines the theoretical energy-resolving power of either an ESA or magnet. This is similar to the theoretical mass-resolving power of either a magnetic sector or double-focusing mass spectrometer, which can be calculated from eq 2. In both equations, $A_{y}$ is the dispersion of the given sector, $A_{\mathrm{X}}$ is the image magnification, $S_{\mathrm{O}}$ is the sector object slit width, $S_{1}$ is the sector image slit width, and $\Delta$ is the total image aberration of the system ([18], and Cody, R. C., JEOL USA, Inc., private communication):

$$
\begin{aligned}
& R_{\mathrm{E}}=\frac{E}{\Delta E}=\frac{A_{\gamma}}{A_{X} S_{\mathrm{O}}+S_{\mathrm{I}}+\Delta} \\
& R_{\mathrm{M}}=\frac{M}{\Delta M}=\frac{A_{\gamma}}{A_{\mathrm{X}} S_{\mathrm{O}}+S_{\mathrm{I}}+\Delta}
\end{aligned}
$$

For example, Table 1 lists the ion optical parameters of the JEOL (Peabody, MA) JMS-SX102/SX102A/E five-sector tandem mass spectrometer (Figure 1). Assuming negligible image aberrations ( $\leq 3 \mu \mathrm{m}$; Cody, R. C., JEOL USA, Inc., private communication), the parameters may be used in eqs 1 and 2 to calculate the theoretical energy- and mass-resolving powers of each sector and the double-focusing arrangement at different slit settings.

There are several important consequences of eqs 1 and 2. One is that for the ion beam image width to equal the image slit width, eq 3 must be fulfilled for each sector and for the double-focusing arrangement. Another is that single-sector and double-focusing resolving powers are maximum for maximum ion transmission when eq 3 is fulfilled. The most important consequence in terms of energy-resolving power is the relationship between eq 3 and the energy-resolving powers of the sectors in a two-sector instrument. For any double-focusing mass spectrometer (BE or EB), the energy-resolving powers of the individual magnet and ESA (calculated from equation 1) are equal when the widths of $S_{O}$ and $S_{1}$ are set to fulfill eq 3 . This fact does not change regardless of the setting of the intermediate $\beta$ slit. This means that narrowing the $\beta$ slit increases the energy-resolving powers of both individual sectors equally.

$$
S_{\mathrm{I}}=A_{\mathrm{X}} S_{\mathrm{O}}
$$

One purpose of this article is to demonstrate experimentally the fundamental concepts described in the preceding text. Another purpose is to discuss the meaning of the "energy resolution of the main beam" in terms of the energy-resolving power of the ESA versus the kinetic energy distribution of the main beam. We also show the importance of the energy-resolving slits for correct measurement of the kinetic energy distribution of the precursor ion beam.

\section{Experimental}

\section{Description of the Instrument}

All experiments were performed by using a JEOL JMS-SX102/SX102A/E $\quad B_{1} E_{1} / B_{2} E_{2} / E_{3} \quad$ (MS1/MS2/ MS3) five-sector tandem mass spectrometer (Figure 1). The instrument is comprised of two SX102 doublefocusing instruments followed by a JEOL HX110 ESA. The SX102 was designed using the TRIO program by Matsuda [18] to give minimal second-and third-order double-focusing image aberrations. It has a field arrangement of $\mathrm{qqBqE}$ (quadrupole, quadrupole, homogeneous magnetic sector, quadrupole, cylindrical electric sector). The purpose of the first two quadrupole lenses $\left(Q_{1(1)}\right.$ and $Q_{2(1}$, for MS1 and $Q_{1(2)}$ and $Q_{2(2)}$ for MS2 in Figure 1) is to maximize transmission through a narrow magnet gap and to obtain a very small image magnification. The quadrupole lenses focus the ion beam in the z-axial (vertical, or direction of magnetic field) direction and defocus it in the $y$-axial (horizontal) direction. The result is a narrow beam profile in the $z$-axial direction and a wide beam profile in the $y$-axial direction in the magnet gap. The magnet refo-

Table 1. Ion optical parameters of the JEOL JMS-SX102/SX102A / E five-sector tandem mass spectrometer

\begin{tabular}{lccll}
\hline Sector & $\begin{array}{c}\text { Dispersion }\left(A_{\gamma}\right)^{a} \\
(\mu \mathrm{m})\end{array}$ & $\begin{array}{c}\text { Magnification } \\
\left(A_{x}\right)\end{array}$ & $\begin{array}{c}\text { Object slit } \\
\left(S_{\mathrm{O}}\right)\end{array}$ & $\begin{array}{c}\text { Image slit } \\
\left(S_{1}\right)\end{array}$ \\
\hline \hline$\overline{\left.\mathrm{B}_{1} \text { (and } \mathrm{B}_{2}\right)}$ & 178,100 & 0.118 & $S_{\mathrm{O} 1}$ (and $\left.S_{\mathrm{O} 2}\right)$ & $\beta_{1}$ (and $\left.\beta_{2}\right)$ \\
$\mathrm{E}_{1}$ land $\left.\mathrm{E}_{2}\right)$ & 304,400 & 1.709 & $\beta_{1}$ (and $\left.\beta_{2}\right)$ & $S_{\mathrm{C} 1}$ (and $\left.S_{\mathrm{C} 2}\right)$ \\
$\mathrm{E}_{3}$ & 371,500 & 0.7064 & $S_{\mathrm{C} 2}$ & $S_{\mathrm{C} 3}$ \\
$\mathrm{~B}_{1} \mathrm{E}_{1}$ (and $\left.\mathrm{B}_{2} \mathrm{E}_{2}\right)$ & 304,400 & 0.202 & $S_{\mathrm{O} 1}$ (and $\left.S_{\mathrm{O} 2}\right)$ & $S_{\mathrm{C} 1}$ (and $S_{\mathrm{C} 2}$ ) \\
\hline
\end{tabular}

The dispersion constants are rounded to four significant figures by the manufacturer. It should be noted, however, that in a double-focusing mass spectrometer, the dispersion of the magnet must be equal to the dispersion of the ESA divided by the magnification of the ESA. Consequently. the dispersion of $B_{1}, 178,100$, is equal to $304,400 / 1.709$ rounded off to four significant figures. 


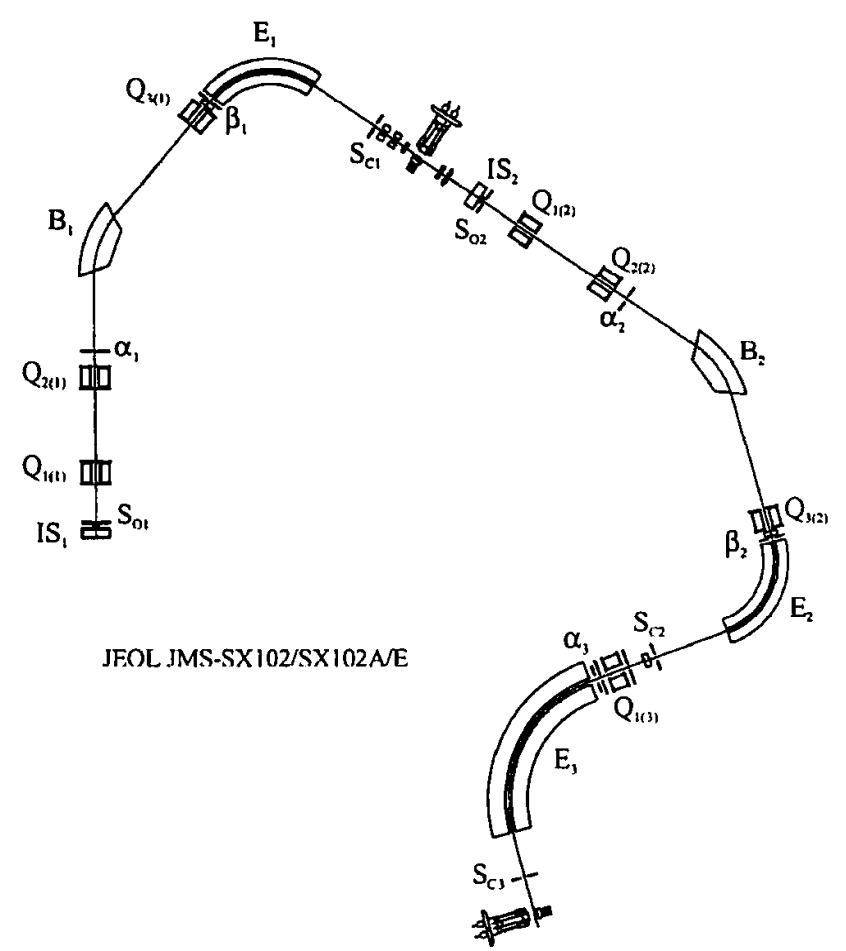

Figure 1. Ion optics of the JEOL JMS-SX102/SX102/E.

cuses the beam in the $y$-axial direction at the $\beta$ slit to give an image magnification of 0.118 . The third quadrupole lens between the magnet and ESA $\left(Q_{311}\right.$ for MS1 and $Q_{3(2)}$ for MS2 in Figure 1) is used to obtain stigmatic or $z$-axial focusing $[18,19]$. The only virtual image is of the ion-source object slit, an image that is formed preceding the magnet by the action of the first two quadrupole lenses.

The addition of a large JEOL HX110 ESA $\left(\mathrm{E}_{3}\right)$ to the commercially available, four-sector SX102/SX102A (Figure 1) provides for some fundamental experiments that can be performed only by using a three-sector $\mathrm{BE} / \mathrm{E}$-type instrument. Relevant to this work is that both $E_{2}$ and $E_{3}$ can be scanned together to obtain a spectrum of the main beam that is transmitted through the fourth field-free region between $B_{2}$ and $E_{2}$. In cases in which the final collector slit $\left(\mathrm{S}_{\mathrm{CB}}\right)$ is fully open, $\mathrm{E}_{3}$ serves as a simple ion guide. Conversely, a scan of $E_{3}$ independent of $E_{2}$ provides for a kinetic energy analysis of a double-focused main beam, which is doublefocused at the collector slit between $E_{2}$ and $E_{3}\left(S_{C_{2}}\right)$. Transmission of an ion beam from MS1 through MS2 is approximately $60 \%$. Approximately $100 \%$ transmission is maintained through $\mathrm{E}_{3}$ by its large radius and bore and by the presence of a final quadrupole lens $\left(Q_{1(3)}\right)$ that is used for stigmatic ( $z$-axial) focusing of the ions. The ion optical parameters-dispersion and magnification - of each sector are given in Table 1.

The SX102/SX102A/E has a series of fully adjustable slits that are relevant in this work: ion-source object slit ( $\mathrm{S}_{\mathrm{O} 1}$ for MS1 and $\mathrm{S}_{\mathrm{O} 2}$ for MS2), $\alpha$ slit $\left(\alpha_{1}\right.$ for MS1 and $\alpha_{2}$ for MS2), intermediate energy-resolving $\beta$ slit ( $\beta_{1}$ for MS1 and $\beta_{2}$ for MS2), and collector-image slit $\left(S_{\mathrm{C} 1}\right.$ for MS1 and $\mathrm{S}_{\mathrm{C} 2}$ for MS2; Figure 1). There is also a final collector slit $\left(\mathrm{S}_{\mathrm{C} 3}\right)$ that follows $\mathrm{E}_{3}$. Each sector thus has an object and image slit, as does the double-focusing arrangement (Table 1). The purpose of the $\alpha$ slit is to reduce the angular spread of the ion beam prior to entering the magnet. Adjustment of the $\alpha$ slit is important to obtain high double-focusing mass resolution because it reduces higher-order image aberrations.

An important feature of the SX102/SX102A/E is the ability to move all collision cells out of the ion beam. The cells in the second, fourth, and fifth field-free regions are raised via pneumatic valves; the cell in the third field-free region is contained in a probe and is manually withdrawn. Removal of the collision cells reduces pressure in the field-free regions and eliminates all interfering slits that could restrict the ion beam. All experiments here were conducted with the collision cells removed from the ion beam. Consequently, the only slits in the second, fourth, and fifth field-free regions are the energy-resolving $\beta_{1}$ and $\beta_{2}$ slits and $\mathrm{S}_{\mathrm{C} 2}$, respectively (Figure 1 ).

\section{Methods}

Benzonitrile molecular ions of $\mathrm{m} / \mathrm{z} 103$ were formed by $70-\mathrm{eV}$ electron ionization in the ion source of MS1. All spectra were obtained by acquisition of narrow 2-s scans across the main beam of ions formed in the ion source by using analog filtering of $100 \mathrm{~Hz}$. Spectra are sums of 5-60 scans.

Experiments that use MS1 and detector 1 as a BE instrument involved holding all scanning parameters constant except for one. The experiments involved either scanning $B_{1}$ while the accelerating and $E_{1}$ voltages are held constant, scanning $E_{1}$ while $B_{1}$ and the accelerating voltage are held constant, or scanning the accelerating voltage while $B_{1}$ and $E_{1}$ are held constant.

"Triple-sector" experiments were performed by passing the molecular ions through MS1 $\left(B_{1} E_{1}\right)$ at a double-focusing resolution of 1000 (10\% valley definition). The ions then were transmitted through MS2 $\left(B_{2} E_{2}\right)$ set at various double-focusing resolutions as described in the text. Ions were detected by detector 2 . Experiments involved either scanning $E_{2} E_{3}$ together while the ion-source accelerating voltage and $B_{2}$ were held constant, scanning $E_{3}$ while the accelerating and $E_{2}$ voltages and $B_{2}$ were held constant, or scanning the accelerating voltage while $E_{2}, E_{3}$, and $B_{2}$ were held constant. In these experiments, the interface region between MS1 and MS2 was tuned before a series of experiments was begun and subsequently was not changed.

\section{Results and Discussion}

There are conflicting definitions of "mass resolution," "mass-resolving power," "energy resolution," and 
"energy-resolving power" in the literature. Consequently, we define these terms with basis in the ionoptical definitions of Bainbridge and Jordan [11], Barber et al. [14], Nier and co-workers [17], Duckworth and co-workers [20], and Wollnik [21]:

1. Mass-resolving power $=M / \Delta M=$ theoretical resolving power with basis in ion-optical design (equation 2).

2. Mass resolution $=\Delta M / M$.

3. Energy-resolving power $=E / \Delta E=$ theoretical resolving power with basis in ion-optical design (equation 1).

4. Energy resolution $=\Delta E / E$.

We also define "realizable resolving power" in accord with Bainbridge and Jordan [11] as observable resolving power determined by an experimental measurement.

\section{BE Mass Spectrometers, ESA Scans, and Realizable Energy-Resolving Power under Double-Focusing Conditions}

Several different experiments were performed by using double-focused MS1 $\left(B_{1} E_{1}\right)$ to compare theoretical to realizable mass- and energy-resolving powers. The experiments involved scans of either the magnetic field, the ESA, or the accelerating voltage. The ionsource object and collector-image slit widths were set to fulfill eq 3 . Different $\beta_{1}$ slit settings were used to give different theoretical energy-resolving powers for the ESA and magnet.
Magnet and ESA scans give the same results (Table 2). At different theoretical double-focusing massresolving (column 2, Table 2) and energy-resolving (column 3, Table 2) powers, scans of the magnetic field while the accelerating and ESA voltages are held constant give the double-focusing mass-resolving power (column 4, Table 2). Likewise, scans of the ESA while the accelerating voltage and magnetic field are held constant give the double-focusing mass-resolving power (column 5, Table 2). These results are entirely in accord with the principles of double-focusing mass spectrometers discussed in the Introduction.

Scans of the accelerating voltage while the magnetic field and ESA voltage are held constant is the method by which researchers traditionally measure the kinetic energy distribution of the main beam in ion'kinetic energy spectrometry (IKES) experiments [1, 22, 23]. (Scans at a constant $\mathrm{B}^{2} / \mathrm{E}$, which give the same results as accelerating-voltage scans, also can be used [24].) IKES experiments are used in EB double-focusing mass spectrometers to study first field-free region metastable ion decompositions. Here, accelerating-voltage scans (holding $\mathrm{B}$ and $\mathrm{E}$ constant) give realizable (measured) energy-resolving powers that reflect the theoretical energy-resolving power of the ESA (and magnet), at least in cases in which the ESA object $(\beta)$ slit is not fully illuminated by the ion beam (numbers with a superscript " $\mathrm{e}$ " in column 6 , Table 2 ). This means that the ion beam is narrower than the width of the $\beta$ slit so that accelerating-voltage scans give flat-topped peaks. In cases in which the $\beta$ slit is fully illuminated (data for $\beta$ slit width of $60 \mu \mathrm{m}$ in column 6 , Table 2), the realizable energy-resolving power of the ESA is less than theoretical. This is because the peaks, which are

Table 2. Theoretical and realizable resolving powers obtained by magnet, ESA, and accelerating-voltage scans across the main beam using $B_{1} E_{1}$

\begin{tabular}{|c|c|c|c|c|c|c|c|}
\hline \multicolumn{3}{|c|}{$\begin{array}{c}\text { Experimental } \\
\text { slit settings }(\mu \mathrm{m})\end{array}$} & \multirow{2}{*}{$\begin{array}{l}\text { Theoretical } \\
\text { double-focusing } \\
\text { resolving power }\end{array}$} & \multirow{2}{*}{$\begin{array}{c}\text { Theoretical } \\
\text { energy- } \\
\text { resolving power }\end{array}$} & \multicolumn{3}{|c|}{$\begin{array}{c}\text { Realizable } \\
\text { resolving power }\end{array}$} \\
\hline$s_{01}$ & $\beta_{1}$ & $S_{\mathrm{Cl}}$ & & & $\begin{array}{c}\text { Magnet scan } \\
10 \% \\
\end{array}$ & $\begin{array}{c}\text { ESA scan } \\
10 \% \\
\end{array}$ & $\begin{array}{c}\text { Voltage scan } \\
10 \% \\
\end{array}$ \\
\hline \multirow[t]{4}{*}{733} & 2000 & 147 & $1000^{d}$ & 85 & 1100 & 1,100 & $80^{e}$ \\
\hline & 1000 & & & 160 & 1100 & 1,100 & $150^{e}$ \\
\hline & 160 & & & 720 & 1400 & 1,400 & 440 \\
\hline & 60 & & & 1200 & 1400 & 1,400 & 570 \\
\hline \multirow[t]{4}{*}{244} & 2000 & 49 & $3100^{d}$ & 88 & 3300 & 3,200 & $90^{e}$ \\
\hline & 1000 & & & 170 & 3200 & 3,000 & $160^{e}$ \\
\hline & 160 & & & 940 & 3200 & 3,000 & 690 \\
\hline & 60 & & & 2000 & 3200 & 3,000 & 1000 \\
\hline \multirow[t]{4}{*}{73} & 2000 & 14 & $11000^{d}$ & 89 & 8200 & 10,000 & $110^{\mathrm{e}}$ \\
\hline & 1000 & & & 180 & 9000 & 10,000 & $170^{e}$ \\
\hline & 160 & & & 1100 & 9000 & 10,000 & $1000^{e}$ \\
\hline & 60 & & & 2600 & 9000 & 10,000 & 2200 \\
\hline
\end{tabular}

${ }^{a}$ Resolving power calculated from widths of peaks at $10 \%$ height.

${ }^{b}$ Calculated from eq 2 and ion optical parameters for $B_{1} E_{1}$ (Table 1 ).

Calculated from eq 1 and ion optical parameters for $E_{1}$ (Table 1). These numbers can also be obtained from eq 1 and ion optical parameters for $B_{1}$.

"For theoretical double-focusing mass-resolving powers of 1000,3000 , and 10,000, the $\alpha_{1}$ slit width is $12,000,8000$, and $2000 \mu \mathrm{m}$, respectively.

The peak is flat-topped. 
approximately Gaussian in shape and wider than the $\beta$-slit width, reveal the kinetic energy distribution of the precursor ions, which obscures the measurement of the true energy-resolving power of the ESA (and magnet). (The issue of energy-resolving power versus kinetic energy distribution is discussed in more detail in a later section.)

There are four important points to be made about the data in Table 2-points that are true for doublefocused $\mathrm{BE}$ and $\mathrm{EB}$ instruments.

1. ESA scans give the double-focusing resolving power of the instrument.

2. Under conditions in which the ion source and collector slit widths fulfill eq 3 , accelerating-voltage scans give the energy-resolving power of the individual ESA and magnet, as discussed in the Introduction. Thus, realizable energy-resolving powers for flat-topped peaks reflect the theoretical energyresolving powers of the magnet.

3. Accelerating-voltage scans reveal the kinetic energy distribution of precursor ions that enter the first sector, not precursor ions that enter the second sector. This is because at a constant accelerating voltage, the intermediate energy-resolving $\beta$ slit only passes ions that have a finite range of kinetic energies to the second sector. Thus, acceleratingvoltage scans only give a maximum value for the kinetic energy distribution of the precursor ion beam that enters the second sector.

4. The intermediate $\beta$ slit width has a significant impact on energy-resolving power: At high doublefocusing resolving powers, an open $\beta$ slit results in poor energy-resolving power.

\section{"Triple-Sector" Experiments and ESA Scans}

Some of the foregoing results are substantiated and further understood from experiments that use MS2/MS3 $\left(B_{2} E_{2} / E_{3}\right)$ of the JEOL JMS-SX102/ $S X 102 A / E$ (Figure 1). The main beam formed in ion source 1 is transmitted through MS1 set at a doublefocusing mass-resolving power of 1000 according to equation $3\left(\beta_{1}=2000 \mu \mathrm{m}\right)$. MS2 is set at a theoretical double-focusing mass-resolving power of 11,000 according to eqs 2 and 3 . The $\beta_{2}$ slit of MS2, between $B_{2}$ and $E_{2}$, is fully open to $2000 \mu \mathrm{m}$. These slit settings give $B_{2}$ and $E_{2}$ theoretical energy-resolving powers of 89 according to eq 1 .

The first experiment reiterates that scans of the ESA of a $B E$ instrument measure double-focusing resolving power. Here, $S_{\mathrm{C}_{3}}$, which is after $E_{3}$, is set fully open to $5000 \mu \mathrm{m}$ so that $E_{3}$ operates only as an ion guide. (This means that the energy-resolving power of $E_{3}$ is less than either $B_{2}$ or $E_{2}$, and $E_{3}$ has no function in the experiment except to guide the ions to the detector.) $E_{2}$ and $E_{3}$ are then scanned together. The scan of $E_{2} E_{3}$ across the precursor ion gives a realizable (measured) resolving power of 8600 ( $10 \%$ height; Figure $2 \mathrm{~A}$ ). This
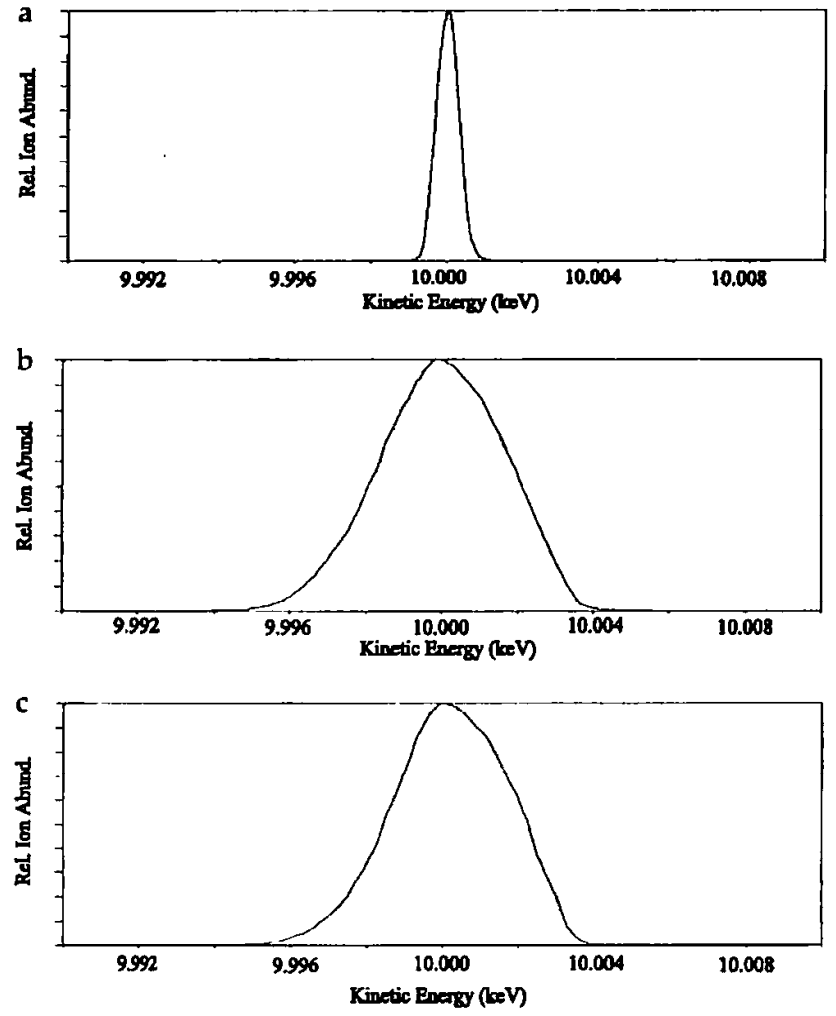

Figure 2. Results from scanning either $E_{2}(A), E_{3}(B)$, or the accelerating voltage $(C)$ across the main beam by using $B_{2} E_{2} / E_{3}$. Slit settings were (A) $S_{\mathrm{O}_{2}}=73, \alpha_{2}=2000, \beta_{2}=2000, S_{\mathrm{C} 2}=14$, $S_{C 3}=5000 \mu \mathrm{m}$; (B) same as in (A) except $S_{C 3}=20 \mu \mathrm{m}$; (C) same as in (B).

resolving power reflects the double-focusing mass-resolving power of $B_{2} E_{2}$ as expected from principles of double-focusing mass spectrometers.

The peak in Figure 2A shows neither the energy-resolving power of $E_{2}$ (or $B_{2}$ ) nor the kinetic energy distribution of the main beam. A "triple-sector" experiment, however, reveals the actual kinetic energy distribution of the main beam. MS2 and MS3 are used as a tandem $B_{2} E_{2} / E_{3}$ instrument in which the main beam is double-focused by $B_{2} E_{2}$ prior to kinetic energy analysis by $E_{3}$. Holding all other conditions from Figure $2 \mathrm{~A}$ the same, $\mathrm{S}_{\mathrm{C} 3}$ is now closed to $20 \mu \mathrm{m}$, and $\mathrm{E}_{3}$ alone is scanned. Under these conditions, the theoretical energy-resolving power of $E_{3}$ is 14,000 . The kinetic energy spectrum of the double-focused main beam (Figure 2B) gives a peak that is significantly wider than in Figure 2A. The spectrum in Figure $2 B$ is a spectrum of the kinetic energy distribution of the main beam, which gives a realizable energy-resolving power of 1400 and $\Delta E$ of $7.1 \mathrm{eV}$ ( $10 \%$ height). Here again the kinetic energy distribution of the main beam obscures the measurement of the true energy-resolving power. (The issue of energy-resolving power versus kinetic energy distribution is discussed in more detail in a later section.)

Another experiment that uses MS2/MS3 as a tandem $B_{2} E_{2} / E_{3}$ instrument demonstrates that accelerat- 
ing-voltage scans also give the kinetic energy distribution of the main beam. The experimental conditions are identical to those in Figure 2B, except the accelerating voltage, instead of $\mathrm{E}_{3}$, is scanned. The acceleratingvoltage scans (Figure 2C) give an almost identical kinetic energy distribution, which results in a realizable energy-resolving power of 1600 and $\Delta E$ of $6.2 \mathrm{eV}$ (10\% height).

There are four important points to be made about the data in Figure 2.

1. ESA scans of a double-focusing $B E$ instrument give the double-focusing resolving power.

2. Scans of the last ESA of a triple-sector $(B E / E$ or $E B / E$ ) instrument can correctly provide a measurement of the kinetic energy distribution of the main beam. This is because the ion beam is doublefocused prior to entering the last ESA. Doublefocusing by $B E$ (or $E B$ ) results in precursor ions that enter the last ESA with trajectories that are independent of translational energy (velocity and momentum) and original angle of entrance $(\alpha)$ into the double-focusing $\mathrm{BE}$ (or $\mathrm{EB}$ ) instrument. This means that the ion beam that exits the double-focusing instrument is analogous to an ion beam that exits an ion source. Thus, the last ESA operates independently of $\mathrm{BE}$ (or EB).

3. Accelerating-voltage scans measure the kinetic energy of ions that can be transmitted to the detector. In the experiments in Figure $2 B$ and $C$, the energyresolving power of $E_{3}$ defines the energy-resolving power of the entire $B_{1} E_{1} / B_{2} E_{2} / E_{3}$ instrument. Thus, accelerating-voltage scans give the realizable energy-resolving power of the most highly energy-resolving sector that precedes the detector.

4. Accelerating-voltage scans in triple-sector instruments give the same results as scans of the last ESA as long as there are no kinetic energy restrictions between the first and last sectors.

\section{$B E$ Mass Spectrometers and ESA Scans under Non-Double-Focusing Conditions: Angular Defocusing}

As discussed in the preceding text, the last ESA of a triple-sector $B E / E$ (or $E B / E$ ) mass spectrometer operates entirely independently of $\mathrm{BE}$ (or EB). This is not the case for the ESA of a simple BE mass spectrometer that is operated under double-focusing conditions: Precursor ions enter the ESA with trajectories that are entirely dependent on translational energy and original angle of entrance $(\alpha)$ into the magnet. This is because the velocity dispersion and angular focusing of the magnet directionally focuses ions of different kinetic energies at different points along the plane of the $\beta$ slit. Furthermore, the ESA is intimately linked to the magnet because of its equal and opposite velocity dispersion and directional focusing.
Consequently, the only way to use an ESA of a double-focusing BE mass spectrometer to energyanalyze a precursor ion beam is to eliminate doublefocusing. Thus, the ESA will operate independently of the magnet. One way to accomplish this is by angularly (directionally) defocusing the beam.

The ion beam in the SX102 $\left(B_{1} E_{1}\right)$ can be defocused angularly by detuning the first two quadrupole lenses $\left(Q_{1(1)}\right.$ and $Q_{2(1)}$ in Figure 1). MS1 is set to a doublefocusing mass-resolving power of 5200 according to eqs 2 and 3 . The $\beta_{1}$ slit is closed to $10 \mu \mathrm{m}$, which gives a theoretical energy-resolving power of 6600 for the ESA and magnet. The quadrupole lenses are then detuned (set to $0 \mathrm{~V}$ ).

Results from angular defocusing are significantly different from the preceding results acquired by using a double-focused instrument. Magnet scans (Figure $3 \mathrm{~A}$; mass scale converted to energy scale) reveal a main beam that clearly is not double-focused. It is likewise obvious that the double-focusing mass-resolving power of 5200 is not achieved (realizable mass-resolving power $=132$ ). In particularly sharp contrast to the data from a double-focused instrument, ESA scans (Figure 3B) do not give the same results as magnet scans. Furthermore, accelerating-voltage scans (Figure 3C) now give identical results to ESA scans. ESA and accelerating-voltage scans give the realizable (mea-
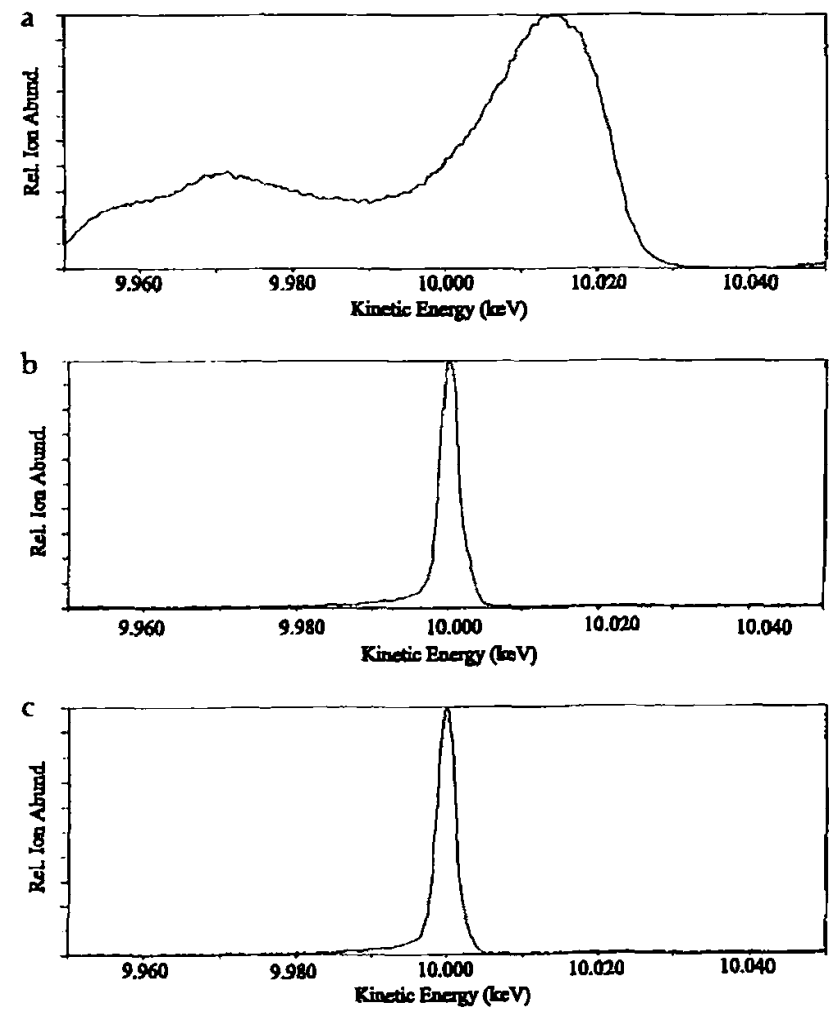

Figure 3. Measurements of realizable energy-resolving power by using $B_{1} E_{1}$ under non-double-focusing conditions afforded by angular defocusing of the magnet: (A) magnet scan; (B) ESA scan; (C) accelerating-voltage scan. Slit settings were $S_{\mathrm{O} 1}=147$, $\alpha_{1}=12,000, \beta_{1}=10, S_{C 1}=29 \mu \mathrm{m}$. 
sured) energy-resolving power of the ESA, which is only $1600 \pm 4 \%$ ( $10 \%$ height) because the kinetic energy distribution of the main beam $(\Delta E=6.2 \pm 0.2 \mathrm{eV}$ at $10 \%$ height) obscures the measurement of true energy-resolving power.

There are two important points to be made about the data in Figure 3.

1. As long as there is no directional focusing, both ESA and accelerating-voltage scans can reveal the kinetic energy distribution of ions that actually enter the ESA. This is because the non-directionally-focused precursor ions impinge upon the intermediate $\beta$ slit with trajectories that are independent of their kinetic energies and $\alpha$.

2. For an EB mass spectrometer, an equal but opposite case would hold. If correct directional focusing by the ESA were thwarted, either accelerating-voltage or magnet scans would give the kinetic energy (momentum) distribution of the main beam that enters the magnet.

\section{BE Mass Spectrometers and ESA Scans under Non-Double-Focusing Conditions: Narrow Source and $\beta$ Slits, Wide Collector Slit}

Another case in which a BE mass spectrometer can be rendered non-double-focusing involves operation of the magnet as a single-focusing sector where the ion source and $\beta$ slits are very narrow but the collector slit is very wide. Under normal double-focusing conditions, the $\beta$ slit would be set very wide, not narrow. Then, the result of a very narrow source slit but a very wide collector slit would be flat-topped peaks in magnet scans: the collector image slit would not be fully illuminated by the ion beam. With a very narrow $\beta$ slit, however, the magnet operates independently of the ESA. The result is that the magnet functions as a single-focusing sector and the ESA only serves as an ion guide for the magnet.

This condition is achieved by setting the ion-source object slit of MS1 $\left(\mathrm{S}_{\mathrm{O} 1}\right)$ to $147 \mu \mathrm{m}$, the $\beta_{1}$ slit to $10 \mu \mathrm{m}$, and the collector-image slit $\left(\mathrm{S}_{\mathrm{Cl}}\right)$ to $940 \mu \mathrm{m}$. This gives a theoretical "double-focusing" mass-resolving power of 310 according to eq 2 . The theoretical energy-resolving powers of the magnet and ESA are not the same, however. The magnet has a theoretical energy-resolving power of 6500; the ESA has 320 . Thus, eq 3 is not fulfilled. Furthermore, the instrument is not a true double-focused mass spectrometer because a change in the accelerating voltage changes the mass-to-charge ratio that is transmitted to the detector, as discussed further in subsequent text.

The result of the preceding slit settings is that magnet scans do not give flat-topped peaks that reflect the "double-focusing" mass-resolving power of 310. Magnet scans instead give the realizable (measured) momentum and thus energy-resolving power of the sin-
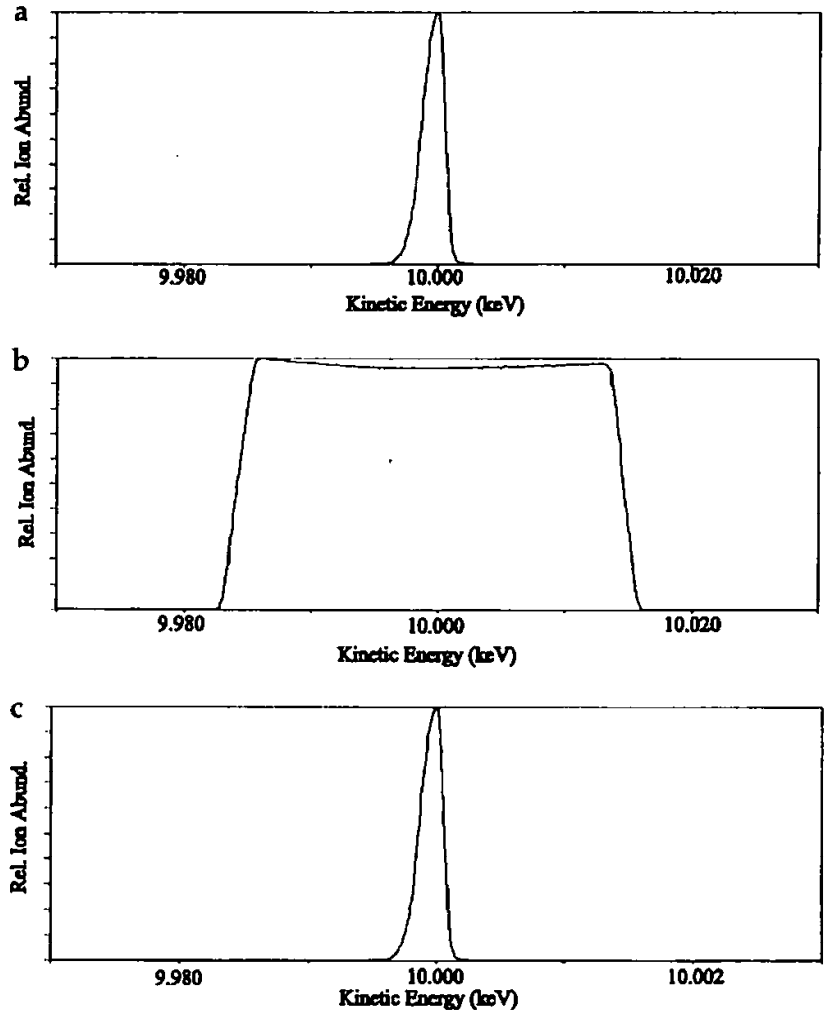

Figure 4. Measurements of realizable energy-resolving power by using $B_{1} E_{1}$ under non-double-focusing conditions afforded by having narrow source and $\beta$ slits and wide collector slit: (A) magnet scan; (B) ESA scan; (C) accelerating-voltage scan. Slit settings were $S_{\mathrm{O} 1}=147, \alpha_{1}=4000, \beta_{1}=10, S_{\mathrm{Cl}}=940 \mu \mathrm{m}$.

gle-focusing magnetic sector (Figure 4A; mass scale converted to energy scale). The peak in Figure $4 \mathrm{~A}$ only gives a realizable momentum (energy) resolving power of 2800 (10\% height) because the kinetic energy distribution of the precursor ions $(\Delta E=3.6 \mathrm{eV}$ at $10 \%$ height) obscures the measurement of true energyresolving power.

Magnet scans are identical to accelerating-voltage scans (Figure 4C); the latter also show that the magnet has a realizable momentum (energy) resolving power of 2800 ( $10 \%$ height). The two types of scans give the same results because the instrument is not truly double-focusing: the magnet functions as a single-focusing sector. Thus, the mass-to-charge ratio that is transmitted to the detector is inversely proportional to $V$ at constant $B^{2}$ (eq 4). This means that at constant magnetic field strength, the product $(m / z \times V)$ remains constant for all mass-to-charge ratio values so that decreases in the accelerating voltage $V$ simply increase the transmitted mass-to-charge ratio. This fact is verified easily by changing the accelerating voltage and watching the shift in mass-to-charge ratio of the peak on an oscilloscope display.

$$
m / z=B^{2} r_{\mathrm{B}}^{2} e / 2 V
$$

ESA scans (Figure 4B) give entirely different results: a realizable resolving power of $310(10 \%$ height). It is 
obvious that the flat-topped peak (Figure 4B) does not show the kinetic energy distribution of the precursor ion main beam. The question becomes whether ESA scans reflect the theoretical energy-resolving power of the ESA (320) or the "double-focusing" mass-resolving power (310). To answer this question, experiments were performed in which the $\beta_{1}$ slit was widened to change the ESA energy-resolving power-experiments that do not, however, affect "double-focusing" massresolving power. Changing the $\beta_{1}$ slit width has no effect on the results shown in Figure 4B. Furthermore, even under correct double-focusing conditions, which include correct angular focusing of the magnet and ion-source object and collector-image slits set to fulfill eq 3, narrowing the $\beta$ slit width has no effect on ESA scans.

These results indicate that ESA scans always reflect the "double-focusing" resolving power of the instrument, unless angular focusing is eliminated. In the experiment shown in Figure 4B, however, the instrument as a whole is not actually double-focused, as demonstrated previously. The magnet, nonetheless, is still velocity dispersing and directionally focusing the ion beam along the plane of the $\beta_{1}$ slit. This means that the ion beam that enters the ESA is dependent on translational energy and angle of entrance $(\alpha)$ into the magnet. Consequently, the ESA functions as it would in a true double-focused instrument, which is to directionally and velocity focus the ions onto the plane of the collector slit.

Opening the ion-source slit wide $(147 \mu \mathrm{m})$ and narrowing the $\beta(10 \mu \mathrm{m})$ and collector $(1 \mu \mathrm{m})$ slits give the ESA a higher theoretical energy-resolving power $(17,000)$ than either the double-focusing massresolving power (9900) or the single-focusing mass(energy-) resolving power of the magnet (6500). These slit settings also do not fulfill eq 3 . These conditions, however, provide for double-focusing: neither the ESA nor the magnet functions as a simple ion guide. Consequently, ESA and magnetic field scans both give realizable resolving powers of 10,000 ( $10 \%$ height), which reflect double-focusing mass-resolving power. Accelerating-voltage scans give a realizable energy-resolving power of 3014 (50\% height), which reflects the kinetic energy distribution of the main beam that enters the magnet.

There are four important points to be made about the foregoing data.

1. Under conditions of correct angular focusing through the magnet, ESA scans always give the double-focusing resolving power of the instrument.

2. Magnet scans under conditions in which the magnet functions as a single-focusing sector give the kinetic energy (momentum) distribution of precursor ions that enter the magnet, not ions that enter the ESA.

3. Accelerating-voltage scans always give the realizable energy-resolving power of the most highly energy-resolving sector.
4. For an EB mass spectrometer, an equal but opposite case would hold. Magnet scans would always give the double-focusing resolving power of the instrument as long as the ESA was correctly directionally focusing. ESA scans under conditions in which the ESA functioned as a single-focusing sector would give the kinetic energy distribution of precursor ions that enter the ESA. Accelerating-voltage scans would give the same result. Accelerating-voltage scans would always give the realizable energy-resolving power of the most highly energyresolving sector.

\section{Energy-Resolving Power Versus the Kinetic Energy Distribution of the Main Beam: Importance of Energy-Resolving Slits}

The data presented in all the above sections focus on how to correctly employ ESA and accelerating-voltage (or $\mathrm{B}^{2} / \mathrm{E}$ ) scans to measure realizable energy-resolving power of double-focusing and triple-sector mass spectrometers. One issue to which the discussion frequently alludes is whether the realizable energy-resolving power reflects either the true energy-resolving power or the kinetic energy distribution of the main beam. For example, it was mentioned that in Table 2, data that are flat-topped peaks (labeled with a superscript " $e$ ") reflect the true energy-resolving power of the ESA and magnet. In contrast, data that give lower realizable energy-resolving powers than the theoretical instead reflect the kinetic energy distribution of the main beam, which obscures the measurement of true energy-resolving power.

This can be understood from an analogy to mass-resolving power. For a mono-mass beam of ions of $\mathrm{m} / \mathrm{z}$ 1000.0 , the ion-source object and collector-image slits of a double-focusing mass spectrometer may be set to give a theoretical and measurable double-focusing mass-resolving power of 1000 . This resolving power may not be observable experimentally, however, for an ion of $m / z 10,000$ because of unresolved ${ }^{13} \mathrm{C}$ and other isotopes that broaden the experimentally measured peak width $(\Delta M)$. In the latter case, the "mass distribution" obscures the measurement of the true doublefocusing mass-resolving power. A similar circumstance occurs for energy-resolving power. The object and image slits of a perfectly directionally focusing ESA (no higher order image aberrations) may be set to give an instrumentally inherent energy-resolving power of 10,000 . This resolving power may not be observable experimentally, however, because the kinetic energy distribution of the main beam obscures the measurement of the true energy-resolving power.

The most important factor that influences whether one measures either the energy-resolving power of an ESA or the kinetic energy distribution of the main beam is the settings of the energy-resolving slits that immediately precede and follow the ESA. For BE mass 
spectrometers, the slits are the intermediate $\beta$ and collector slits. For triple-sector BE/E (or EB/E) mass spectrometers, the slits are the double-focusing collector slit (after BE or EB) and the final slit after the last ESA. To measure the kinetic energy distribution of the main beam and $\Delta E$, which are the important parameters in MIKES experiments, it is vital that the energyresolving slits be narrow enough and the theoretical resolving power be great enough so that the energy distribution is the limiting factor in realizable energyresolving power.

This concept is most clearly demonstrated from different peak shapes and realizable energy-resolving powers obtained by using different energy-resolving slit widths and a "triple-sector" type of experiment. The ion beam is transmitted through both MS1 and MS2 at double-focusing mass-resolving powers of 1000 , in accord with eq $3\left(\mathrm{~S}_{\mathrm{C} 2}\right.$, which precedes $\mathrm{E}_{3}$, is set to $147 \mu \mathrm{m})$. The $\beta_{1}$ and $\beta_{2}$ slits are fully open to 2000 . The only slit that is changed is $\mathrm{S}_{\mathrm{C} 3}$, which immediately follows $E_{3}$. Scans of $E_{3}$ are performed.

When the final energy-resolving slit $\left(\mathrm{S}_{\mathrm{C} 3}\right)$ is wide $(1000 \mu \mathrm{m})$, a theoretical energy-resolving power of 340 for $E_{3}$ is calculated from equation 1. Experimentally, scans of $E_{3}$ give a flat-topped main beam and a realizable energy-resolving power of 350 (10\% height; Figure $5 \mathrm{~A}$ ). The realizable energy-resolving power is the same as the theoretical because $S_{C 3}$ is not fully illuminated by the ion beam: a $147-\mu \mathrm{m}$ object at $S_{C_{2}}$ gives a $104-\mu \mathrm{m}$ image that is scanned across the $1000-\mu \mathrm{m} \mathrm{S}_{\mathrm{C} 3}$ slit. The peak in Figure 5A does not reveal the kinetic energy distribution of the main beam. The peak only reveals the energy-resolving power of the ESA, which is low. The energy-resolving power of the ESA thus is the limiting factor in realizable energy-resolving power.

The situation changes when $\mathrm{S}_{\mathrm{C} 3}$ is narrowed so that its width is significantly less than the kinetic energy width of the main beam. Closing $S_{C 3}$ to $20 \mu \mathrm{m}$ gives a theoretical energy-resolving power of 3000 , but realizable energy-resolving power is only 1900 (10\% height; Figure $5 \mathrm{~B})$. The peak now shows the kinetic energy distribution and width of the main beam $(\Delta E=5.3 \mathrm{eV}$ at $10 \%$ height), which obscures the measurement of true energy-resolving power. To verify that the spectrum in Figure 5B indeed shows the kinetic energy distribution, another experiment is performed in which $\mathrm{S}_{\mathrm{C} 3}=10 \mu \mathrm{m}$ (theoretical energy-resolving power $=$ 3300 ; Figure $5 \mathrm{C}$ ). The realizable energy-resolving power and $\Delta E$ (1900 and $5.3 \mathrm{eV}$ at $10 \%$ height, respectively) are the same as in Figure $5 \mathrm{~B}$, which verifies that the energy-resolving slits in the experiment in Figure $5 \mathrm{~B}$ were narrow enough to correctly give the kinetic energy distribution of the precursor ion beam. In Figure $5 B$ and $C$, the kinetic energy distribution of the main beam is the limiting factor in realizable energy-resolving power.

This same concept is demonstrated for simple BE instruments. MS1 is used at a double-focusing massresolving power of 5200 (eq 3 is fulfilled). One set of
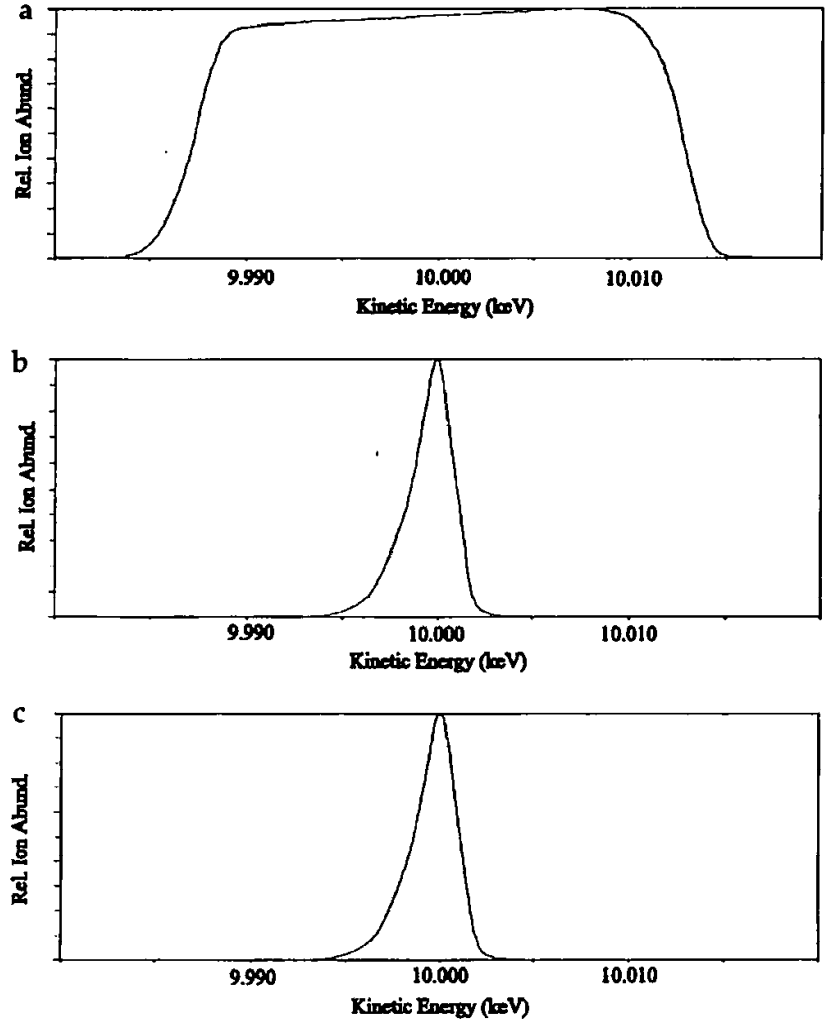

Figure 5. Measurements of realizable energy-resolving power by using different $S_{C 3}$ slit widths and scanning $E_{3}$ of $B_{2} E_{2} / E_{3}$. Slit settings of $S_{\mathrm{C} 3}$ were (A) 1000, (B) 20, and (C) $10 \mu \mathrm{m}$. Other slit settings were $S_{\mathrm{O} 2}=733, \alpha_{2}=12,000, \beta_{2}=2000, S_{\mathrm{C} 2}=$ $147 \mu \mathrm{m}$.

data from accelerating-voltage scans is acquired at a theoretical ESA energy-resolving power of $3100\left(\beta_{1}=\right.$ $40 \mu \mathrm{m}, \mathrm{S}_{\mathrm{Cl}}=29 \mu \mathrm{m}$; Figure $6 \mathrm{~A}$ ). Another set is acquired at a theoretical ESA energy-resolving power of $6600\left(\beta_{1}=10 \mu \mathrm{m}, \mathrm{S}_{\mathrm{C} 1}=29 \mu \mathrm{m}\right.$; Figure 6B). Despite the large change in theoretical energy-resolving powers, the realizable energy-resolving powers and $\Delta E$ are identical $(2400 \pm 3 \%$ and $4.2 \pm 0.1 \mathrm{eV}$ at $10 \%$ height, respectively). The peaks in both experiments show the kinetic energy distribution of the precursor ion beam that enters the magnet, which obscures the measurement of true energy-resolving power. Thus, to correctly measure the kinetic energy distribution of the precursor ion and $\Delta E$, the intermediate $\beta$ slit must be narrow enough so that it is fully illuminated by the ion beam.

There are two important points to be made about the foregoing data.

1. The most important factor that influences whether one measures either the energy-resolving power of an ESA or the kinetic energy distribution of the main beam is the setting of the two energy-resolving slits that immediately precede and follow the ESA. For BE mass spectrometers, the slits are the intermediate $\beta$ and collector-image slits. For EB mass spectrometers, the slits are the ion-source ob- 

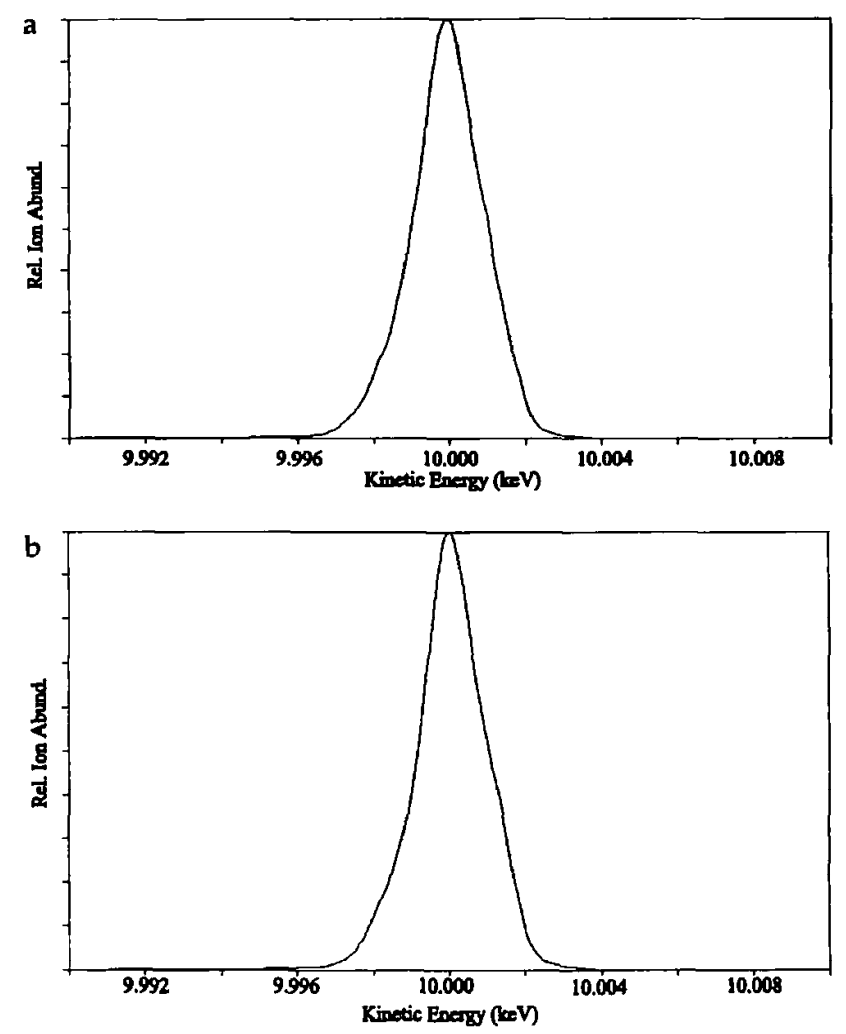

Figure 6. Measurements of the kinetic energy distribution of the main beam and $\Delta E$ by using $B_{1} E_{1}$ and accelerating-voltage scans at different theoretical energy-resolving powers: (A) $S_{() 1}=$ 147, $\alpha_{1}=4000, \beta_{1}=40, S_{\mathrm{Cl}}=29 \mu \mathrm{m}$; (B) slit settings same as in (A) except $\beta_{1}=10 \mu \mathrm{m}$.

ject and intermediate $\beta$ slits. For either $B E / E$ or $E B / E$ instruments, the slits are the double-focusing collector (after BE or EB) and final collector (after the last ESA) slits.

2. To measure the kinetic energy distribution of the main beam and $\Delta E$, it is vital that the preceding energy-resolving slits be set so that the theoretical energy-resolving power of the ESA is not the limiting factor in realizable energy-resolving power.

\section{Conclusions}

Our data demonstrate that the use of ESA scans to measure the "energy resolution of the main beam" in double-focused BE mass spectrometers is not in accord with principles of double-focusing. Under doublefocusing conditions, scans of the ESA neither reveal the energy-resolving power of the ESA nor the kinetic energy distribution of the main beam. Consequently, the "energy resolution" and main beam widths previously reported [1-9] from ESA scans simply would be the double-focusing mass resolution and mass widths, respectively, if the instruments were operated under double-focusing conditions.

The only way to use a BE mass spectrometer and ESA scans to measure the realizable energy-resolving power of the ESA is to operate the instrument under conditions in which there is no directional focusing. Thus, the instrument is not double-focused. This approach, however, may overestimate the kinetic energy distribution of the main beam because the ions may enter the ESA at such large angles that angular aberrations broaden the peak.

Under any instrumental condition, the validity of the use of ESA scans and BE mass spectrometers to measure the kinetic energy distribution of the main beam can be determined by comparing the results to results from magnet scans. If a peak width measured by ESA scans was approximately the same as measured by magnet scans at the same peak height, the ESA scans would be giving the double-focusing resolving power, not the kinetic energy distribution.

The kinetic energy distribution and $\Delta E$ can be measured by using either accelerating-voltage or $B^{2} / E$ scans. For double-focused BE or EB mass spectrometers, accelerating-voltage (or $\mathrm{B}^{2} / \mathrm{E}$ ) scans, however, only measure the kinetic energy distribution of the ion beam that enters the first sector. Thus for $B E$ instruments, accelerating-voltage (or $\mathrm{B}^{2} / \mathrm{E}$ ) scans only give a maximum for the kinetic energy distribution of the main beam that enters the ESA. Furthermore, in BE, $E B$, and multisector (three-, four-, five-sector) instruments, accelerating-voltage (or $B^{2} / E$ ) scans always measure the realizable energy-resolving power of the most highly energy-resolving sector. Dependent on slit settings, this may not be an ESA.

The energy-resolving power of an ESA and the kinetic energy distribution of the main beam cannot be measured simultaneously. To measure a realizable energy-resolving power that is equal to the true energyresolving power of the ESA, energy-resolving power must be poor so that the kinetic energy distribution of the main beam does not obscure the measurement. Conversely, to measure the kinetic energy distribution of the main beam, energy-resolving power must be high so that the energy distribution is the limiting factor in realizable resolving power. Thus, there is no way to actually measure the "energy resolution of the main beam."

It is important in measurements of KERDs that the realizable energy-resolving power be limited by the kinetic energy distribution of the main beam, not by the energy-resolving power of the ESA. As a result, the peak shape of the main beam, as determined by accelerating-voltage (or $\mathrm{B}^{2} / \mathrm{E}$ ) scans in either $\mathrm{BE}$ or $\mathrm{EB}$ mass spectrometers, correctly reflects its maximum possible kinetic energy width $(\Delta E)$. To guarantee this condition, the energy-resolving slits that immediately precede and follow the ESA must be narrowed to the point at which changes in their widths do not affect the measured peak width. For triple-sector mass spectrometers, the double-focusing collector slit and the slit immediately following the last ESA must likewise be narrowed to the point at which changes in their widths do not affect the measured peak width. 


\section{Acknowledgments}

We acknowledge the National Science Foundation (CHE-9113272) and the Petroleum Research Fund (25280-AC), which is administered by the American Chemical Society, for funding, and the NIH (1S10RR06276) and NSF (CHE-9119862) for the use of the JEOL JMS-SX102/SX102A/E as a shared instrument. We also acknowledge Don Rempel (Washington University), Fumio Kunihiro (JEOL Ltd., Japan), Bob Bateman (VG Inc., England), Jean Futrell (University of Delaware), and Mike Bowers (UCSB) for helpful discussions.

\section{References}

1. Cooks, R. G.; Beynon, J. H.; Caprioli, R. M.; Lester, G. R Metastable lons; Elsevier Scientific Publishing: Amsterdam, 1973; pp 61-63, 73 .

2. Terwilliger, D. T.; Beynon, J. H.; Cooks, R. G. Proc. Roy. Soc. London Ser. A 1974, 341, 135.

3. Morgan, R. P.; Beynon, J. H.; Bateman, R. H.; Green, B. N. Int. J. Mass Spectrom. Ion Phys. 1978, 28, 171.

4. Amaya, A. M.; Mead, W. L.; Brenton, A. G.; Proctor, C. J.; Beynon, J. H. Int. J. Mass Spectront. Ion Phys. 1980, 36, 57.

5. Jarrold, M. F.; Illies, A. J.; Bowers, M. T. Chem. Phys. 1982, 65 , 19.

6. llies, A. J.; Jarrold, M. F.; Bowers, M. T. I. Am. Chem. Soc. 1982, 104, 3587.

7. Rumpf, B. A.; Derrick, P. J. Int. J. Mass Spectrom. Jon Processes 1988, 82, 239.

8. van Koppen, P. A. M.; Jacobson, D. B.; Illies, A.; Bowers, M. T.; Hanratty, M.; Beauchamp, J. L. J. Am. Chem. Soc. 1989, 111, 1991.

9. Vékey, K.; Pócsfalvi, G. Org. Mass Spectrom. 1992, 27, 1203.

10. (a) Herzog, R. Z. Phys. 1934, 89, 447; (b) Mattauch, J.; Herzog, R. Z. Phys. 1934, 89, 786; (c) Herzog, R. Z. Naturforsch. 1953, $8 a, 191$.
11. Bainbridge, K. T.; Jordan, E. B. Phys. Rev. 1936, 50, 282.

12. Johnson, E. G.; Nier, A. O. Plyss. Rev. 1953, 91, 10.

13. (a) Hintenberger, H.; König, L. A. Z. Naturgforsch. 1957, 12n, 140; (b) Hintenberger, H.; König, L. A. Z. Naturgforsch. 1957, $12 n, 443$.

14. Barber, R. C.; Bishop, R. L.; Duckworth, H. E.; Merdith, J. O.; Southon, F. C. G.; Van Rookhuyzen, P.; Williams, P. Rev. Sci. Instrum. 1971, 42, 1.

15. Swann, W. F. G. J. Franklin Inst. 1931, 212, 439.

16. Bleakney, W. Am. Phys. Teacher 1936, 4, 12.

17. (a) Nier, A. O.; Roberts, T. R. Phys. Rev. 1951, 81, 507; (b) Quinsenberry, K. S.; Scolman, T. T.; Nier, A. O. Phys. Rev. 1956, 102, 1071; (c) Nier, A. O. In Nuclear Mass and Their Determination; Hintenberger, H., Ed.; Pergamon Press: London, 1957; pp 194-201.

18. Matsuda, H. Int. I. Mass Spectrom. Ion Processes 1985, 66, 209.

19. (a) Matsuda, H.; Matsuo, T.; Fujita, Y.; Sakurai, T.; Katakuse, I. Int. I. Mass Spectrom. Ion Processes 1989, 91, 1; (b) Ewald, H.; Liebl, H.; Sauermann, G. In Nuclear Mass and Their Determination; Hintenberger, H., Ed.; Pergamon Press: London, 1957; pp 194-201.

20. (a) Duckworth, H. E; Barber, R. C.; Venkatasubramanian, V. S. Mass Spectroscopy, 2nd. ed.; Cambridge University Press: Cambridge, England, 1986; (b) Duckwortl, H. E.; Ghoshah, S. N. In Mass Spectrometry; McDowell, C. A., Ed.; McGrawHill: New York; 1963; Chap. 7.

21. Wollnik, H. Optics of Charged Particles; Academic Press: New York, 1987; p 99.

22. Cooks, R. G.; Kim, K. C.; Keough, T.; Beynon, J. H. Int. I. Mass Spectrom. Iont P/yys. 1974, 15, 271.

23. Jones, E. G.; Bauman, L. E.; Beynon, J. H.; Cooks, R. G. Org. Mass Spectrom. 1973, 7, 185.

24. (a) Boyd, R. K.; Beynon, J. H. Org. Mnss Spectrom. 1977, 12 , 163; (b) Haddon, W. F. Alnal. Chem. 1979, 51, 983. 